\title{
Effect of texture and grain shape on anisotropy
}

\author{
G.R. Canova
}

LPMM, Faculté des Sciences, Université de Metz, Ile du Saulcy, 57045 Metz Cedex, France

(Reçu le 26 mai 1987, révisé le 15 août 1987, accepté le 22 septembre 1987)

RESUME :

Les différentes approches visant à modéliser l'anisotropie de matériaux polycristallins ont été résumées. Au niveau du monocristal, la formulation utilisant 1a loi de Schmid ou la viscoplasticité montre clairement l'anisotropie microscopique due au durcissement et aux changements d'orientation. On montre aussi que 1a viscoplasticité conduit à une rénartition unique des glissements cristallographiques indépendemment de la sensibilité à la vitesse. Les propriétés macroscopiques sont obtenues à partir des propriétés microscopiques au moyen de modèles qui sont des images de 1 'interaction grain-matrice. On montre que des modèles à basses interactions devraient être utilisés en grandes déformations. On montre explicitement comment la forme des grains affecte 1 'interaction. Finalement, les surfaces de charge sont prédites et on montre que des grains plats engendrent des singularités sur la surface de charge globale.

ABSTRACT :

An attempt is made to summarize the different ways of modelling the anisotropy of a polycrystalline material. At the single crystal level, the formulation based on Schmid law or viscoplasticity shows clearly the microscopic anisotropy due to the hardening and the orientation changes. It is also shown how viscoplasticity leads to a unique slip system activity irrespectively of the rate sensitivity. Macroscopic properties are derived from the microscopic ones via models that are a picture of the grain-matrix interaction. It is shown that low interaction models should be investigated for large strain descriptions. It is also shown how grain shapes explicitely affec the interaction. Finally, yield surfaces are predicted, and it is demonstrated that flat grain shapes can induce vertices on the overall yield surface.

Most industrial applications dealing with e.g. metal forming (at low and high strain rates) like deep drawing, or simply the rolling operation have to deal with a preexisting or induced anisotropy which affects the material behavior and wich can be associated with some problems (earing, plastic instabilities...) or with some properties that the engineer wants to optimize.

In this summary paper, we want to assess the description of the anisotropy of a polycrystalline material. The overall material anisotropy is, first of all, affected by the single crystal behavior. The Schmid law is assumed to hold in the following treatment and is used to describe the preexisting anisotropy of the single crystal. The induced anisotropy can be affected by different microscopic hardening laws and by the lattice rotations. Both effects will be described. 
A model is needed to link the microscopic behavior to the macroscopic behavior. This model is based on the "picture" made of the relation between the grain and its neighbourhood. Different classes of models will be presented, namely static and Taylor models as well as different classes of self-consistend models. The problem of the choice of the active slip systems is important inasmuch as the texture evolution depends on it. That problem is particularly accute when that choice is ambiguous. We will show the influence of the viscosity on the selection. The effect of the grain-shapes on the grain-neighbourhood interaction will also be described both in an extreme model, the Relaxed Constraint Theory, and a modified Kröner model based on elastic grain-matrix interactions.

A careful analysis of large strain tex ture predictions particularly in uniaxiail compression and rolling of f.c.c. materials using both the Full and Relaxed Constraints Theories can help us determining in which direction to improve thr models.

Finally a method will be outlined for building yield surfaces and plastic potentials. Some results will be shown demonstrating the effect of textures and grain shapes. Some yield surfaces will be drawn and examined with respect to flow stability in given mechanical tests.

\section{THE SINGLE CRYSTAL ANISOTROPY}

We are dealing with a crystalline material having a plastic deformation along well defined slip systems characterized by slip planes of normal $n$ s ( $s$ refers to the slip system) and of slip direction $b^{\mathbf{s}}$. The basic statement used in the description of the single crystal behavior is the Schmid law (1) i.e. that a resolved shear stress on a given slip system needs to reach a critical value in order for a dislocation on that system to move promoting plastic straining. The Critical Resolved Shear Stress (CRSS) cannot be exceeded in quasi-static conditions. This basic assumption can be expressed as :

$$
m_{i j}^{s} s_{i j}=\tau_{c}^{s}
$$

with $m_{i j}^{s}=b_{i}^{s} n_{j}^{s}$

and $S$ is here the stress deviator tensor; the double summation convention will be used throughout this paper. The LHS of equ. (1) is unaffected by a hydrostatic pressure, since :

$$
\operatorname{tr}(m)=n_{i} b_{i}=0
$$

the slip direction and the slip plane normal being orthogonal. In this approach no information is given concerning the shear rate $\dot{Y}^{s}$ of the slip systems; they are indeterminate unless further assumptions are made namely concerning the work-hardening rate.
A slight modification of the assumption (1) can be made when the material is no longer taken as rate-insensitive. In this alternative way we state that the rate of plastic shearing along given slip systems is proportional to the resolved shear stress at a power characteristic of the race-sensitivity :

$$
\dot{\gamma}^{S} / \dot{\gamma} O=\left(\tau^{S} / \tau \stackrel{S}{0}\right)^{m}
$$

where $m^{\prime}=1 / m, m$ being the rate sensitivity parameter. ypical values of $\mathrm{m}^{\prime}$ are bet ween 20 and 100 in the cold deformation range for most materials. $\tau_{0}^{s}$ and $\dot{\gamma} 0$ are respectively the reference shear stress (RRSS) and the shearina rate associated to it. $\tau$ o is not the CRSS.

The velocity gradient due to the activity of one slip system deforming at a shearing $\mathrm{s}$ can be expressed

$$
L_{i j}=b_{i}^{s} n_{j}^{s} \dot{\gamma}^{s}
$$

so that the strain rate due to the contribution of all systems is written as :

$$
D_{i j}=\left(m_{i j}^{s}\right) \dot{\gamma} s
$$

where $(m)$ is the symmetrical part of the tensor $m$. Since $\operatorname{tr}(m)=0$, then $\operatorname{tr}(D)=0$, i.e. the material volume remains constant. Equ.(1) could have been written as :

$$
\left(m_{i j}^{s}\right) S_{i j}=\tau_{c}^{s}
$$

since $S$ is a symmetrical tensor. The latter equation represents a hyperplane in the 50 stress space whose normal is $(\mathrm{m})$, and the polyhedron built up by applying equ. (1) to all given slip systems will be made out of vertices, $4 \mathrm{th}$, 3rd and 2 nd order edges and planes. Five independent slip systems are needed to build a vertex, and four, three and two systems respectively to make the subsequent stress states. The derivative of eq. (1') with respect to the stress components is the strain rate, i.e. :

$$
D_{i j}=\dot{\gamma}^{s} \partial f / \partial s_{i j}
$$

where the function $f$ is equ. (1). This shows that normality is obeyed by the Schmid $\mathrm{law}$ and therefore the vield. surface and the plastic potential coincide.

For a viscoplastic material, equs.(3) and (5) can be combined to give :

$$
\eta_{i j}=\left(m_{i j}^{s}\right)\left[\left(m_{k \ell}^{s}\right) s_{k \ell}^{\prime} / \tau_{0}^{s}\right]^{m^{\prime}}
$$

A viscoplastic potential of the form :

$$
f=\left[\tau_{0}^{s}\left(m_{i j}^{s} s_{i j} / \tau_{0}^{s}\right)^{m^{\prime}+1}\right] /\left(m^{\prime}+1\right)
$$


can be built so that equ. (5) can be considered as the normality rule. Equ. (8) is not associated with the concept of vield but rather with the one of flow, therefore the term "flow surface" is more appropriate here. On fig. 1 are shown the potentials corresponding to the Schmid law and to materials of increasing rate sensitivity. It can be seen, as has been exposed by Kocks (2) that the shapes are very similar for low values of the rate sensitivity, but that there is no more singularities as soon as the material has a non-zero rate sensivity parameter. The importance of this effect will be demonstrated more clearly in the forthcoming section.

Knowing the slip systems, their resolved shear stress and the rate sensitivity parameter (if necessary), it is possible to construct a (visco-) plastic potential characteristic of the single crystal anisotropy. This surface is referred to a given frame and depends upon two terms : the tensors $\left(m^{s}\right)$ and the constants $\tau_{c}^{s}\left(o r \tau_{0}^{s}\right)$. In the crystal reference frame the former ones are known and constant but change with the orientation when e.g. a fixed external frame is considered. The latter set of terms are influenced by the microscopic hardening mechanisms. At a given time, both types of terms have integrated the previous history and the yield (flow) surface describes therefore the state of the anisotropy. The induced anisotropy at the single crystal level is due to the variation of those two terms, i.e. texture development and microscopic hardening.

Calling $\mathbb{W}^{*}$ the rate of orientation change, the rate of change of the tensors $\left(\mathrm{m}^{\mathrm{s}}\right), \mathrm{can}$ be expressed as :

$$
\dot{m}^{s}=w^{*} \cdot m^{s}-m^{s}-w^{*}
$$

it is noticeable (as intuitively expected) that the tensors $\left(\dot{m}^{s}\right)$ are orthogonal to $\left(\mathrm{m}^{s}\right)$, and that $w^{*}$ induces a "rigid-body rotation" of the yield (flow) surface, i.e. its shape is kept invariant, only its orientation has changed (fig.2a).

It is most commonly assumed that the rate of change of the set of CRSS's is linearly related to the shearing rates along the slip systems:

$$
\dot{\tau}_{c}^{s}=H_{s s^{\prime}} \dot{\gamma}^{\prime}
$$

where $H$ represents the hardening matrix. For viscoplastic materials, it is commonly assumed that $\boldsymbol{r}_{0}^{s}$ (the RRSS) changes in the same way and homothetically to $\boldsymbol{\tau}^{S}$ (the CRSS). According to Franciosi (3), H can have different structures depending on what types of dislocation interactions are considered as important. When all terms are equal isotropic hardening of the slip systems is assumed which keeps the CRSS's on all systems equal at any time and all varying with time. The yield (flow) surface shape is not affected, only its size is changing. When $H$ no longer stays isotropic, the set of CRSS's (RRSS's) does not remain uniform (if it were uniform to start with) and the yield (flow) surface shape changes (fig.2b). Having roughly outlined the single crystal anisotropy, the next step consists in trying to derive the polycrystal anisotropy. The tool for that purpose is a texture model.

\section{TEXTURE MODELLING}

The purpose of all texture models is to derive (or assume) what. happens to a constituent crystallite embedded in a polycrystal whose boundary conditions are given. Hill (4) has shown that all models lie in between two assumptions : in the first one, the Sachs assumption (5), the stress is uniform throughout the polycrystal, enforcing strict equilibrium, and entirely relaxing compatibility conditions : in the second one, the Taylor assumption (6), the strain rate is taken as uniform, enforcing total compatibility between grains, and entirely neglecting equilibrium and internal stresses. In between are all types of self-consistent models where neither the strain rate field nor the stress field are uniform troughout the polycrystal. We will refer here to some elastoplastic models and to some viscoplastic models as well.

2.1 The Sachs model

Stress field uniformity is here assumed,

i.e. :

$$
\stackrel{\sigma}{\sim}=\sum
$$

where $\sigma$ ana $z$ are respectively the local and the macroscopic stress tensors. When this equality is used in a strict way (i.e. equality in norm and direction), only few grains are active simultaneously, since the stress will fall either inside or outside the yield surface of all the grains, only for few exceptions will it fall on the yield surface. Equ. (11) is usually applied in "direction" and not in "norm", i.e. the stress norm is allowed to deviate from grain to grain so that, in general, only one single slip system is activated per grain. When the material is rate-insensitive, the amount of microscopic shear strain on the active slip system is derived from work-hardening considerations. The authors (38) who used this model (in different ways) find that, particularly in rolling; the Brass type texture is found (fig. 3 ). One may therefore think that this model is an indirect way of deriving textures of a material exhibiting latent hardening, twinning..., i.e. a highly anisotropic material.

\subsection{Taylor models}

The explicit assumption expressed by Taylor (6) is that the strain (or strain rate) field is uniform troughout the polycrystal, i.e.:

$$
d=D
$$

where $d$ and $\mathcal{L}$ are respectively the local and macroscopic strain rates. Five systems are therefore necessary to solve those five independent equations, using equ.(5). Since many choices of five independent systems are available to solve those equations, the final choice is the one(s) minimizing the plastic work-rate

$$
\dot{W}_{1}=\tau_{c}^{s} \dot{\gamma}
$$

Chin and Mammel (7) have shown that the active systems are the same if chosen in the dual way proposed by Bishop and $\mathrm{Hill}(8)$. 
In this alternative formulation, the systems should be in the Schmid conditions, and maximize the work-rate: $\quad i_{2}=s_{i j} d_{i j}$

here $S$ is the local stress tensor. Both problems lead to the same answer, i.e. :

$$
\dot{W}_{1}=\dot{W}_{2}
$$

and the slip systems are the same. When the microscopic shears are calculated, the orientation changes are derived by stating that the local total rate of rotation equals the macroscopic. This rotation is the one associated to the local convected coordinates transformed by the local displacement gradient (or the velocity gradient when a rate form is used). The lattice rotation represents the orientation change with respect to the sample frame, and the so called plastic rotation is the rotation associated with the change of the convected coordinates assuming an invariant lattice. The "total" rotation is the sum of both the latter rotations, i.e.:

$$
\underline{w}^{\top}=w^{*}+w \underline{P}
$$

The plastic spin is easily calculed knowing the microscopic shears from the skew-symmetric part of equ. (4), the orientation changes are therefore derived with the help of equ.(16). The implicit assumption in the Tayl or type theories is in fact the uniformity of the velocity gradient field. Some predictions have been performed by several authors $(9,10,11,12)$, and the results agree at least qualitatively with the existing final orientations found in aluminum and copper (figs.4) which brought a great success to that model.

Van Houtte (13) tried to find the Brass rolling texture by incorporating twinning as proposed by Chin et al.(14). He found that an unrealistic twin volume fraction was necessary in order to get the desired texture. Several authors also noted some systematic deviations compared to real textures, namely in torsion, rolling and compression : in the first case, Cohen and Montheillet (15) found experimentally on aluminum that the (111) partial fiber texture vanished at the profit of the (100) (011) component. In rolling, Honneff and Mecking (16) noticed that the Taylor component was very strong and not in agreement with the real textures. Finally the compression case is also of interest, since predicted textures deviate from reality, but will be studied in more detail in a forthcomina section.

The Taylor approach is very similar to the Lin approach (17), which including elasticity, assumes uniformity of the total strain. Asaro and Needleman (18) derived an elastoviscoplastic formulation of the Lin's hypothesis, which do not bring much difference compared to strict Taylor results. This fact will be explained further in the self-consistent section.

When a material exhibits isotropic hardening of the slip system, the solution of the Taylor or the Bishop/Hill problem is not unique in the sense that more than five systems can be picked up, fulfilling all conditions. The non-uniqueness of the slip system selection is not onlv encountered when five systems are needed but also when four and three independent systems are required.

It can therefore also affect self-consistent schemes. before summarizing briefly those types of modelizations, the ambiguity problem will now be adressed.

\subsection{The ambiguity problem}

When all CRSS's of the single crystal are equal, and a strain-rate is applied to it, the maximum work principle will select a stress state (usually a vertex of the single crystal yield surface, when five strain rate components are prescribed) which brings more than five systems in the Schmid conditions. Table 1 lists all vertices of an f.c.c. single crystal with the associated slip systems. In that particular case either six or eight systems are potentially active. When only four or even three strain rate components are known, the stress states associated will be respectively of the fourth order or of the third order.

Tables 2 and 3 show that also in those cases, the ambiguity remains.

When different sets of microscopic shear rates are solution of equ.(5), any barycenter of those solutions is also a solution, since equ. (5) is a linear set of equations, meaning that there is in principle an infinite number of solutions. Bishop (9) evaluated the importance of this ambiauity on the texture develnoment, by considerina the extre me cases, i.e. all possible sets of five systems on Fin. 5 shows, in uniaxial compression, what is the error range in the orientation change due to the ambiguity. It is particularly noticeable that the error is minimum close to the (111) orientation but extreme close to (100) and (110).

Different ways of treating the ambiguity problem have been proposed in the past. The most widely used has been the averaging technique, which consists into making an arithmetic average of all plastic spins obtained by all choices of five systems. Van Houtte (13) proposed to pick up at random one set of five systems, arguing that, in fact the CRSS's are not exactly all equal but slightly "vibrating" about a known value, so that the vertices split up into many vertices each having five systems and no more. His results do no differ significantly from the ones of the averaging technique, when the calculations are done on a polycrystal. If a single crystal is simulated, however, particularly in a region of high ambiruity, like (110), it is conceivable that the orientation may go back and forth. An alternative way is also used, which has been derived by Renouard and Winterberger (19) : it consists in minimizing the second order plastic work rate. It is here stated that, when the slip systems have a choice, they will try to decrease as much as possible the texture hardening. This method has given encouraging results on partially constrained single crystals (20), but seems to fail for polycrystals in simple cases like tension and compression particularly in domains of large ambiguities (21). When the viscoplasticity is used, it is possible to invert numerically equ. (7) in order to find the applied deviatoric stress, which in turn leads unambiguously to the microscopic shear rate repartition, equ. (3). 
A simple standard Newton-Raphson procedure can be used safely, since a viscoplastic potential can be built up leading to a unique stress-strain rate relationship. If a given strain rate is applied to a crystal of decreasing rate sensitivity, Table 4 , it is worth noting that when $m$ decreases below a value of about 0.05 , the number of active systems stays the same, and the microscopic shear repartition also reaches a stable set of values. The ambiguity problem arises only when $m$ is strictly equal to zero, but vanishes as soon as $m$ slightly departs from zero. When $m$ is greater than 0.1 ; , however, more systems are active as has been shown by Canova et al.(22). Since the shear rate repartition is unique and stable for low $m$ values, it can be stated that the calculated set of $s$ also holds by continuity for $m=0$. The results do not differ greatly from the ones obtained using the averaging technique as shown in figs.6.

This ambiguity problem has brought some difficulties even in self-consistent models that will now be briefly summarized.

\subsection{SELF-CONSISTENT MODELS}

The first self-consistent model used is due to Kröner who used the Eshelby solution of the elastic interaction between an inclusion and an infinite elastic matrix surrounding it. Knowing the local compliance matrix $\mathcal{C}$ of the material, it is possible to relate the stress to the elastic strain by the relation :

$$
\underset{\sim}{\alpha}=\underset{\sim}{c}: \varepsilon^{\mathrm{e}}
$$

where $C$ is here, for simplicity, assumed not to depend on the location and $\varepsilon^{e_{i s}}$ the (small) elastic strain. The equilibrium conditions can be written :

$$
\sigma_{i j, j}=0
$$

assuming that $\underset{\sim}{C}$ is homogeneous, equ. (18) can be rewritten as :

$$
c_{i j k \ell} u_{k, \ell j}+f_{i}=0
$$

i.e. Navier equations, where $\vec{u}$ is the total displacement vector, and $\vec{f}$ is a body force expressed as :

$$
f_{i}=\left(c_{i j k \ell}\right.
$$

Those equations can be solved using the Green functions which are solution of the set of linear equations :

$$
c_{i, j k \ell} f_{k m, \ell j}\left(r-r^{\prime}\right)+\delta_{i m} \delta\left(r-r^{\prime}\right)=0
$$

The knowledge of those functions enables us to calculate the displacement field at any location $r$ with the equation :

$$
u_{i}=U_{i}+\int_{r_{i j}}\left(r-r^{\prime}\right) f_{j}\left(r^{\prime}\right) d r^{\prime}
$$

The displacement 'gradient field is obtained by making the derivative with respect to the coordinates $x$ :

$u_{i j}=u_{i j}+\int r_{i n, j m}\left(r-r^{\prime}\right) c_{n m k \ell}\left(r^{\prime}\right) \varepsilon_{k}^{p}\left(r^{\prime}\right) d r^{\prime}(23)$ rrom tnis, equation, it is possible to first symmetrize :

$\varepsilon_{i j}^{\top}=E_{i j}^{\top}+\int \Gamma_{i j m n}\left(r-r^{\prime}\right) C_{m n k \ell}\left(r^{\prime}\right) * \varepsilon_{k \ell}^{p}\left(r^{\prime}\right) d r^{\prime}$ and then separate the elastic strains from the plastic strains. Using the definition of the Eshelby tensor :

$$
S_{i j m \bar{n}}=C_{i j k l} \int \Gamma_{k t m n}\left(r^{\prime}\right) d r^{\prime}
$$

it is possible to use equs.(24) and (25) with (17) to get the interaction formula :

$J_{i j}-\sum_{i j}=C_{i j k \ell}\left(I_{k \ell m n}-S_{k \ell m n}\right)\left(E_{m n}^{p}-\varepsilon_{m n}^{p}\right)$ (26) which-sets up the differences between the local stresses and plastic strains. The latter equations are obtained by discretizing the medium into two parts : the grain and the infinite matrix. The tensor s results from an integration over the grain volume of the tensor $C:[$. This tensor therefore depends also on the grain shape, since the integration domain changes with the grain shape. This point will be more developed in a forthcoming section. The tensor $I$ is the fourth rank identity tensor, $E^{p}$ and $\varepsilon^{P}$ are the macroscopic and local plastic strains. It can be noted that both the stress-strain relation and the orientation changes are included in equ. (23), since the symmetrical part leads to the interaction equation and the antisymmetrical part leads to the definition of the local total rotation $W^{\top}$ which controls the orientation changes with help of equ.(16). Here again, the grain shape will affect the results (as will be shown later) since the volume integral of the antisymmetrical part of $G$ depends on the integration domain, i.e. the grain shape. The Kröner interaction formula is a simplification of equ. (27) for isotropic elasticity and a Poissnn's ratio of about $1 / 3$, in this case, we get : $\quad \sigma_{i j}-\Sigma_{i j}=\mu\left(E_{i j}^{p}-\varepsilon_{i j}^{p}\right)$

Hill (23) derived also a self-consistent scheme based on the formulation of the grain behavior :

$$
d \sigma_{i j}=L_{i j k \ell}^{c} d \varepsilon_{k \ell}^{p}
$$

He ends up, following the same reasoning, with an interaction formula :

$$
d \sigma_{i, j}-d \sum_{i j}=L_{i j k \ell}\left(d E_{k}^{p}-d \varepsilon_{k \ell}^{p}\right)
$$

where $L^{*}$ is theinteraction tensor deduced by an implicit inteyral equation :

$$
L^{*}=\left\langle\underset{\sim}{L}:\left(L_{\infty}^{C}+\underline{L}\right)^{-1}:\left(\underline{L}+\underline{L}^{*}\right)\right\rangle
$$

Here $<>$ designates the volume average, $L$ and $L$ are the tangent tensors used in equ.(27) respectively for the inclusion and the matrix.

Zaoui and Berveiller (24) derived a simplified form of Hill's model by scaling the compliance matrix $c$ in Kröner theory with a parameter $\propto$ which changes the Kröner interaction formula in the following form :

$$
\underline{\sigma}-\underset{\sim}{\Sigma}=\alpha \mu\left(\underline{\sim}^{p}-\underline{\varepsilon}^{p}\right)
$$

the term $\alpha$ is an elastoplastic interaction term. Altough the term $\alpha$ is fictitious, its variation enables us to "scale" all models from Sachs to Taylor. When $\alpha=0$, equ. (30) leads to the Sachs condition, whereas when $\alpha$ is infinite, it leads to the Taylor condition. On fig. 7 is drawn the slip systems activity as a function of the term $\alpha \mu / H$, where $H$ is the hardening rate. It is clear that tne kröner, Lin and Taylor models lead to the same slip sustem activity, and that the Sachs hypothesis constitutes a special case (only one active system). 
There is a transition region, however, that can be investigated by self-consistent schemes, where none of the extreme models would apply. This graph also points out an interesting fact, namely that a slight deviation from strict Sachs assumption is likely to lead to significant different results, which is not the case for Tayl or hypothesis. Recently, Lipinski et al.(25) proposed an alternative way of treating the large strain self-consistent problem, based on the formulation of the elastoplastic behavior of the single crystal derived by Hill and Rice (26), Asaro (27) and Nemat-Nasser (28), solving the equilibrium equations in terms of the nominal stress rate and assuming some symmetry of the tangent behavior of the matrix.

Another approach, due to Molinari et al.(29), assumes a viscoplastic behavior of the single crystal. The equation :

$$
\stackrel{s}{\sim}=\underset{\sim}{A}(D): \underset{\sim}{D}+\underline{\sim}^{\circ}
$$

represents tne tangent behavior of the grain, where $A$ and $S^{\circ}$ can be calculated directly from equ. (7) once the latter one is solved. Obviously both $A$ and $S^{\circ}$ depend on $D$ (or $S$ ) so that equ. (31) is pseudo-linear. The way of treating the problem self-consistently is similar to the approaches explained above, except that in each grain both the deviator stress and the hydrostatic pressure are calculated. The scheme leads to a Kröner-like interaction equation :

$$
S_{i j}-\bar{S}_{i j}=K_{i j k \ell}\left(D_{k \ell}-D_{k l}\right)
$$

where the tensor $K$ depends on the viscoplastic properties of the matrix and on the grain shape. Few results will here be studied :

-comparison of the texture results with respect to Sachs and Tayl or hypothesis

-how far is this model from the extreme

ones.

Obviously $K$ must be updated at each step of strain, the following results were derived by assuming equiaxed grain shapes and isotropic viscosity. Figs.8 show, in rolling of an f.c.c. material (exhibiting isotropic hardening) the texture obtained at a von Mises strain of $100 \%$. Although Sachs is well known to generate the Brass component, Tayl or model finds a Copper type texture, with the Tayl or component that does not appear (at least so strongly) in real cases. It is worth noting that the viscoplastic self-consistent scheme finds a Copper type texture, but no Taylor component. Looking at the statistics concerning the stress and strain-rate deviations (in direction and norm) with respect to the macroscopic values figs.9, it is possible to see that the stress direction is very close to the macroscopic one, which is not the case for the stress norm. The strain rate, however, is quite different from the macroscopic strain-rate both in norm and direction. This model seems therefore closer to Sachs than to Taylor, and is likely to be in the transition region depicted by Berveiller (fig.7). That calculation, in agreement with Berveiller's graph, seems to show that a small deviation from Sachs will lead to results closer to Taylor than to Sachs. The results of this viscoplastic self-consistent scheme also seem to show that the Taylor component found in rolling by the Tayl or method may well be an effect of the large grain-matrix interaction assumed implicitly in Taylor-type models. Obviously, the absence of that component is due to self-consistency and not to viscoplasticity, since viscoplastic Taylor models also find the Taylor component of the rolling texture. -Another interesting result concerns the one of uniaxial compression. It has been found that the texture results obtained on compression using Taylor-like models $(18,30)$ differ from the real textures, in the sense that the high intensities do no fall at the $(110)$ orientation but about $10^{\circ}$ away in the (100) direction (fig.10). Hosford (31) has explained that grains close to the (110) orientation want to deform into plane strain, the stress state required to achieve local axisymmetry being too far deviated from the average stress to be attained.

Such a strain-rate deviation from the average strain-rate is possible if a low grain-matrix interaction is allowed in the material. In high interaction models like Taylor, the grain must do what is dictated by the surrouding matrix, and if the simulated grain is not allowed to do what the real grain does, the simulated texture may be different from the real one. In low interaction self-consistent schemes like the one of Molinari et al., such a deviation is permitted. As expected, this "freedom" brings back the texture to the (110) type (fig.10). The statistics on the strain-rate direction show a very strong peak which corresponds precisely to the plane strain direction, fig.ll.

This tends to show that the texture modelling should be performed by simulating properly low grain-matrix interactions. The grain shape effect will be addressed in the following section.

\subsection{GRAIN SHAPE EFFECTS}

After having observed the systematic deviations between the predicted textures and the measured ones in rolling of f.c.c. materials, Honeff and Mecking (16) associated this fact with a possible grain shape effect. Their argument was the following : when a grain is very flat, like after a large rolling strain, some grains may have different shear strains. As shown on fig.12, it can be intuitively said that a difference of a shear strain $\varepsilon_{13}$ between two neighbouring flat grains can be accomodated in a grain volume fraction decreasing as the flatness (i.e. the strain) increases. It means, in particular, that some strain components may simply be relaxed, i.e. be not continuous from grain to grain, due to the fact that this discontinuity does not produce any reaction stress. They modified kröner formula in order to set zero reaction stress component for the corresponding relaxed strain component. They found, fig.12, that the rolling results agree more closely to the real textures. Kocks and Canova (32) generalized this argument in terms of compatibility and equilibrium conditions that have to be respected for the large grain faces only. Their argument, if applied on the case of fig. 12, is :

$$
{ }^{\sigma_{13}}=\sum_{13} ; \sigma_{12}^{\sigma_{23}}=\sum_{23} ; \varepsilon_{11}=E_{11} ; \varepsilon_{22}=E_{22}
$$

where the (three) compatibility and che (two necessary) equilibrium equations of the face 3 are written. This is the main hypothesis of the Relaxed Constraint theory. Kocks and Chandra (33) derived a way of selecting the active slip systems in this mixed boundary conditions case by plotting yield subsurfaces, calculed by cutting the single crystal yield surface by the prescribed stress planes. 
They also showed a method of getting the orientation changes by setting which line(s) and plane(s) are fixed in space. Kocks et al.(12) derived also a faster method of building yield subsurfaces by using a parametric description of the edges of the yield surface. Tiem et al. (34) generalized further the Relaxed Constraint assumption in the following way: Knowing the grain shape by its principal lengths, the volume fraction affected by a discontinuity of a given displacement gradient component (and not a strain component) was estimated. If this volume fraction tends to zero at infinite strain, it will be relaxed. This method enables us to determine which displacement gradient components are continuous, in order to know the local values of that tensor. Comparing the local and the macroscopic tensors, it is possible to derive which are the prescribed strain components, and also the local total rotation. Tiem et al. also calculated the coefficients associated to the generalized Kröner equation (equ.(23)) and found that some terms of the interaction stress modulus tend to zero relaxina therefore the corresponding strain component. Fig. 13 shows a "vectorized" form of the components $C_{i j k e}$ ( $\left.T_{k \ell m n}-S_{k \ell m n}\right)$ for the rolled grains. It can be seen that it agrees well with the Relaxed Constraint hypotheses as an infinite strain limit. The skewsymmetrical part of equ.(23) can be written as :

$$
w_{i j}^{\top}=\Omega_{i j}^{T}+A_{i j k \ell} \Delta \varepsilon_{k \ell}^{p}
$$

where $A$ can also be calculated knowing the elastic properties and the grain shape. When elasticity is isotropic, and grain principal axes coincide with the $1,2,3$, axes, the tensor $A$ depends only on three parameters :

$$
p=A_{1212} ; q=A_{1313} ; r=A_{2323}
$$

Fig.14 snows the variation of those parameters as grain shape evolves, it can be noted again the good agreement with the Relaxed Constraint assumption at infinite strain. Although predicted texture results are better represented in rolling and torsion (fig.15), the compression results do not agree with the reality, fig.16. This can be attributed to the "Hosford" effect as explained in the previous section, which still holds in the flat grain approximation : the RC theory allows the grain to relax two shear strain components, but still prevents it from deforming in a plane strain mode.

Although grain shape effects act as a mechanical law which tends to allow some fluctuations in some strain components, by decreasing some reaction stress moduli, the other strain components are prescribed to be uniform throuahout the polycrystal. The compression test seems to be a good example showing that even the latter condition is too strict, and lower interaction models should be used.

Having briefly summarized the current models that can take into account both the texture and the grain shape to relate the microscopic properties to the macroscopic ones, the next section will finally deal with the yield surface construction and the influence of both these parameters on the macroscopic plastic properties.

\section{THE POLYCRYSTAL YIELD SURFACE}

When the Schmid yield condition is used at the single crystal level, there is not ambiguity on the definition of the yield surface. At the polycrystal level, however, there is no unambiguous concept of yielding, since plastic straining starts in one crystallite and propagatessthrough all of them as the macroscopic deformation increases. This elastoplastic transition can be visualized both experimentally and through models.

on fig. 17 are shown experimental results of M.G.Stout et al.(36) who tested 1100 Al prestrained in torsion at different offset strains. It can be seen particularly that a yield surface defined by a small offset strain of $5.10^{-6}$ is greatly influenced by the internal stresses created by the torsion prestrain, whereas a larger offset strain of $0.2 \%$ seems to erase partly this effect, although a small Bauschinger effect remains. When an elastoplastic approach is used like the one developped by Lipinski et al (25), fig. 18, the yield surface shape changes drastically between $0 \%$ and $0.02 \%$ offset strain, but seems to keep approximately the same shape at larger offset strains.

The concept of yielding can be associated with an engineering definition of an offset strain of $0.2 \%$, which seems to correspond to the point where the yield surface shape is no much more affected by the internal stresses. The yield surface built in those conditions may be useful when different monotonic tests are done on a given material in different straining directions. When a material is subjected to continuous strain path changes (cyclic loading...), the use of such an offset is a matter of debate.

The last part of this study will be restricted to yield surface construction using either FC or RC theories, which correspond therefore to a rather large offset, since by imposing a strain direction, many slip systems are loaded simultaneously in all the grains of the polycrystal. The method used to build the yield surface is, in fact, by contructing a plastic potential. A strain direction is applied to the polycrystal, and the average work is calculated :

$$
W=\langle f(g) \underset{\sim}{S}(g): \underset{\sim}{E}(g)\rangle=\langle(g) \underset{\sim}{S}(g)\rangle: \underset{\sim}{E}
$$

where $E(g)$ is the plastic strain direction of the grain $\widetilde{g}$, associated to its stress $\mathrm{S}(\mathrm{g})$. The texture comes into the term $f(g)$ which represents the proportion of grains having the same orientation as grain g. Equ. (36) is the one of a plane tangent to the yield surface of normal $E$. The strain direction has to be normalized, e.g. by the von Mises norm, in order to be able to compare $W$ for different strain directions.

When FC conditions are applied, all strain components are uniform throughout the polycrystal. When RC conditions, in turn, are taken, only part of the tensor $E$ is continuous, the prescribed components of stress being the ones corresponding to the non-prescribed strain components (35). Defining by $E^{\prime}$ and $E^{\prime \prime}(g)$ the prescribed and relaxed parts of the Tensor $\underset{\sim}{E}$, 
associated respectively to $S^{\prime}(g)$ and $S^{\prime \prime}$, the resulting and imposed stress components, equ. (36) becomes :

$$
W=\left\langle f(g) \mathcal{\sim}^{\prime}(g)\right\rangle: E_{\sim}^{\prime}+S_{\sim}^{\prime \prime}:\left\langle f(g) E_{\sim}^{\prime \prime}(g)\right\rangle
$$

It is therefore possible to derive $W$ both in FC and $\mathrm{RC}$ conditions. It can be noted on equs; (36) and (37) that the value taken by $W$ depend on all effects discussed above, namely :

- the microscopic anisotropy, through $S(g)$ (or $S^{\prime}(g)$ ) ; the grain state of stress under the strain direction $\underset{\sim}{E}$ (or ${ }^{\prime}$ ) depends on its yied surface,

$$
\text { -the geometrical texture which enters }
$$
through the term $f(g)$

-the morphological texture which makes us uses equ. (36) or (37) depending on whether the grains are equiaxed or not.

The notation used to represent yield surfaces is the following due to Lequeu et al.(37). A deviatoric stress tensor $S$ is transformed into a vector defined by :

$$
\begin{aligned}
& s_{1}=\left(s_{22}-s_{11}\right) / \sqrt{2} \\
& s_{2}=\sqrt{2} s_{33} / \sqrt{3} \\
& s_{3}=\sqrt{2} s_{23} \\
& s_{4}=\sqrt{2} s_{13} \\
& s_{5}=\sqrt{2} s_{12} .
\end{aligned}
$$

This notation has many advantages : an orthogonal $5 \times 5$ matrix can be built that rotates that vector corresponding to the tensor rotation, the norm of that vector is the second invariant of the tensor, the scalar product of two of these vectors is equivalent to the twice contracted product of the tensors, and finally the flow rule is valid with the vectors when the yield surface is expressed in terms of the stress vector components.

The effect of a morphological texture alone can be visualized on fig. 19 , where a random grain orientation distribution is taken. The main effect is to create a vertex in the $S_{23}$ direction because the large flat plane is orthogonal to direction 3. If the large planes of the grains were orthogonal to directions 1 or 2 , the vertices would appear respectively along the axes $S_{13}$ an $S_{22}$. This effect can be of importance in particular for the biaxial stretching test. It is known that the flow stability in such a test depends dramatically on the curvature of the yield surface at the loading point. A vertex at this point is a destabilizing factor. On fig 19 , it can be seen on the $\mathrm{S}_{2}-\mathrm{S}_{3}$ projection that the vertex is three dimensional. This comes from the fact that the RC theory does not make any difference between a "pancake shaped" flat grain and a "chocolate bar shaped" flat grain, in both these cases the two shears $\varepsilon_{13}$ and $\varepsilon_{23}$ are relaxed. Knowing that the texture is here isotropic, it possesses at least the 3-fiber symmettry, i.e. the $\left(S_{1}-S_{2}-S_{5}\right)$ yield surface projection is invariant by any rotation about the axis $S_{2}$. More refined methods that can take into account the non-cylindrical symmetry of the flat grains would probably find that the yield surface does not have such a $\mathrm{S}_{\mathbf{2}}$-fiber symmetry, and therefore that the sharp curvature found in $S_{2}$ is not axisymmetric.

The effect of the texture is illustrated in fig.20 a for a polycrystal having a (simulated) sharp rolling texture. Some corners can also be found which can be of importance for practical purposes. In fig. 20b are combined both grain shape and texture effects, where the yield surface shape again changes.

\section{CONCLUSIONS}

A short summary has been made concerning the relation between microscopic anisotropy, grain shapes, textures and macroscopic anisotropy.

The microscopic anisotropy description is based on the Schmid law, or a viscoplastic flow law which tends to the previous on at the rate-insensitive limit. It has been shown that a viscoplastic potential can be built leading to a unique stress-strain rate response, so that the viscoplastic law can be inverted safely when a strain-rate is applied. The induced microscopic anisotropy is assumed to be due to the orientation changes and/or the microscopic hardening mechanisms.

It has been shown that the slip system selection is unique and the microscopic shear repartition stable for low values of the rate-sensitivity parameter (i.e. $m<0.05$ ), so that the rate-insensitive case can be assumed to be the limit of a set of cases at decreasing ratesensitivities and therefore be unambiguous.

A brief review of texture models has been presented. It has been shown that a small grain-matrix interaction model, which is more likely to be close to the equilibrium conditions than to the compatibility conditions finds results closer to Taylor predictions than to Sachs predictions. An analysis of the grain curling phenomenon in f.c.c. compression or b.c.c. tension shows that low interaction models should be used and their results compared. The grain shape effect is explained through. the Relaxed Constraint hypothesis and through a generalized Kröner formalism. It is shown, in particular, that it decreases the reaction stress modulus on given strain components, which tends to relax them. It is also shown that such an effect is not enough to represent the curling phenomenon, and therefore that low reaction stress moduli should be taken into account irrespectively of the grain shapes. The good texture results of a viscoplastic self-consistent scheme obtained particularly in compression and rolling seem to confirm that hypothesis.

Finally, after having described a method of constructing the yield surfaces in the FC and RC conditions, the grain shape and texture effects have been illustrated. It has been shown in particular that flat grains tend to promote vertices on the yield surface that could probably affect the flow stability in certain deformation modes. 


\section{REFERENCES}

(1) E. Schmid, 1928

(2) U.F. Kocks, Met. Trans., vol.1, p.1121

(3) P;Franciosi, Thèse d'état, Université Paris XIII, 1984

(4) R.Hill, J.Mech. Phys.Sol., vol.13, 1965, p.89

(5) G.Sachs, Z.Verein Deut.Ing., vol.72, 1924,p.734

(6) G.I. Tayl or, J.Inst.Met., vol.62,1938, p.143

(7) G.Y.Chin and W.L.Mammel, Trans TMS-AIME, vol.245, 1969, p.1211

(8) J.F.Bishop and R.Hill, Phil.Mag, vol.42,1951, p.414

(9) J.F.Bishop, J.Mech.Phys.Sol, vol.3.1954, p.130

(10) H.J. Bunge "Mathematique Methoden des Textmanalyse", Akademine Verlag, Berlin 1969.

(11) J.Gil Sevillano, P.van Houtte and E.Aernoudt, Progr.Mat.Sci., vol.25,1981

(12) G.R.Canova, U.F.Kocks and J.J.Jonas, Acta.Met.,vol.32, 1984, P211

(13) P.Van Houtte, Acta.Met., vol .26, 1978, p.591

(14) G.Chin, W.F.Hosf ord and D.R.Mendorf, Proc.Roy.Soc., A309, 1969, p.433

(15) M.Cohen and F.Montheillet, rapport ATP CNRS, contrat $n^{\circ} 3136,1979$

(16) H.Honeff and H.Mecking, in Textures of Materials (Gottsein and Lücke eds), Springer, Berlin, 1978, p.265

(17) T.H.Lin, J;Mech.Phys.Sol., vol.5, 1957, p.143

(18) R.J.Asaro and A.Needleman, Acta.Met., vol.33 $n^{\circ} 61985, p .923$

(19) M.Renouard and M.Winterberger, C.R.Acad.Sci., Paris B290,1980, p.403

(20) A.Skalli, J.H. Driverand M.Wintenberger, Mem.Sci Rev.Met.vol 820 1983, p.241

(21) B.Bacroix, PhD McGill University, 1987
(22) G. Canova, C. Fressengeas, A. Molinari and V.F. Kocks, submitted to Acta.Met.

(23) R.Hili J.Mech Phys.Sol., vol.13, 1965, p.8

(24) H.Berveiller and A.Zaoui,C.R. ise colloque GFR, Paris 1980, p.175

(25) P.Lipinski,M.Berveiller and A. Carmaval, gth Int. Conf. on "Structural Mechanics $n$ Reactor Technology", Lausanne 1987

(26) R.Hill and J.R.Rice, J.Mech.Phys.Sol, vol.20,1972, P. 401

(27) R.J.Asaro, Acta.Met., vol.27, 1979, p.445

(28) S.Nemat-Nasser, Inst.J.Sol.Str., vol.15, 1979, p. 155

(29) A.Molinari, G.R.Canova and S.Ahzi, submitted to Acta.Met.

(30) G.R. Canova and U.F.Kocks Proc. ICOTOM7, (Brakman et al. eds), Netherlands Society for Metals, 1984, p.573

(31) W.Hosford, Proc. TMS-AIME, vol.12, 1961, p.230

(32) U.F. Kocks and G.R.Canova, Proc. 2nd Ris Int.Symp. Deformation of Polycrystals, Mechanisms, and Microstructures (N.Hansen ed), 1981, p.135

(33) U.F.Kocks and H.Chandra Acta.Met., vol.309, 1982 , p. 695

(34) S.Tiem, H.Berveiller and G.R. Canova, Acta-Met., vol.34, n०11, 1986, p.2139

(35) U.F. Kocks, G.R.Canova and J.J. Jonas, Acta.Met., vol.31, 1983, p.1243

(36) M.G.Stout,P.L.Martin, D.E.Helling and G.R. Canova, Int.J.of. Plasticity, vol.l, 1985, p.163

(37) Ph.Leqeu, phD Thesis, Mc Gi11 University, 1986.

(38) T. Leffers, RIS $\emptyset$ Report $n^{\circ} 184,1968$

(39) H. Mecking, Proc. ICSMA 5, Aachen, Germany, 1979.

(40) T. Leffers, RIS $\varnothing$ report $n^{\circ} 302,1975$. 



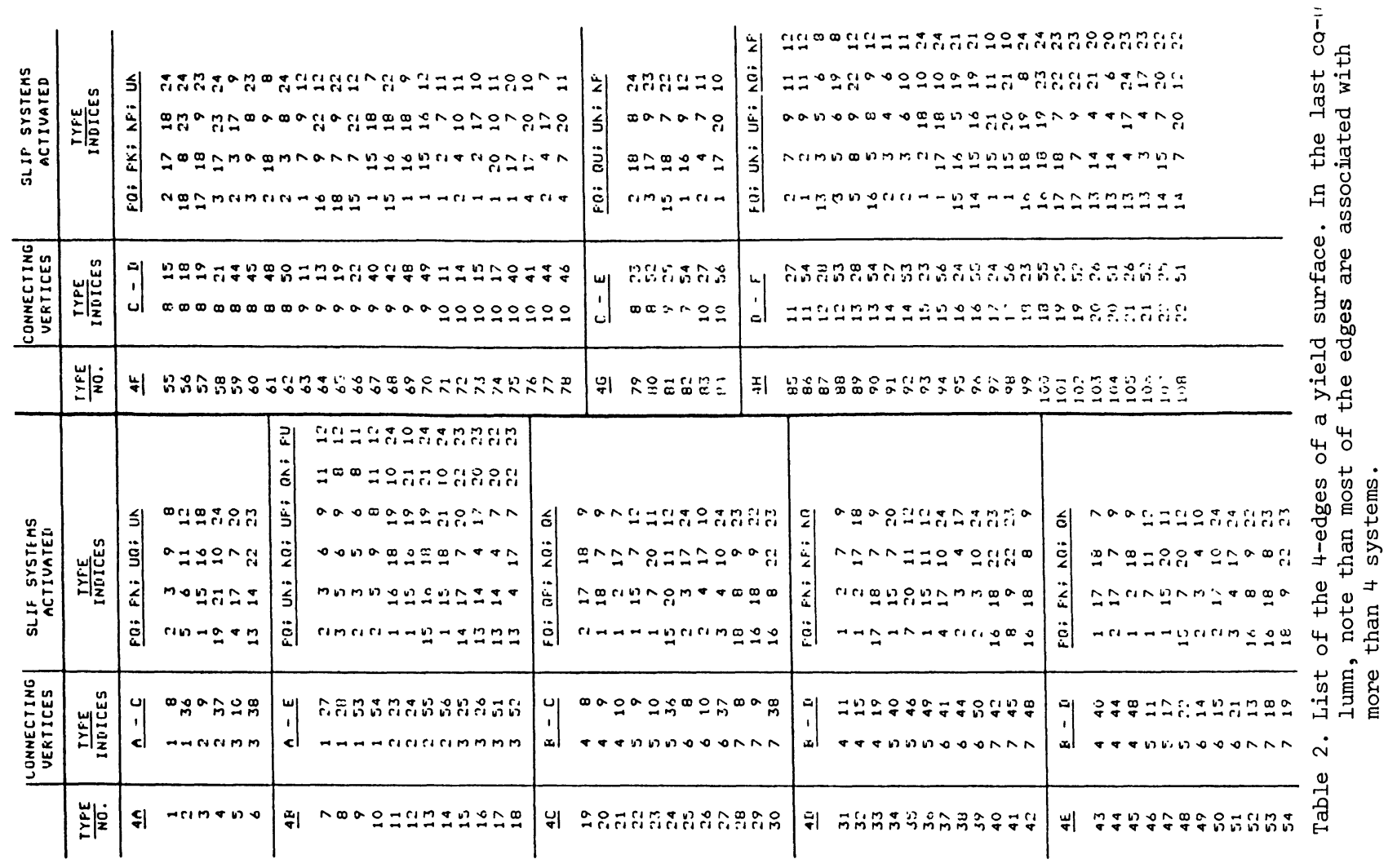

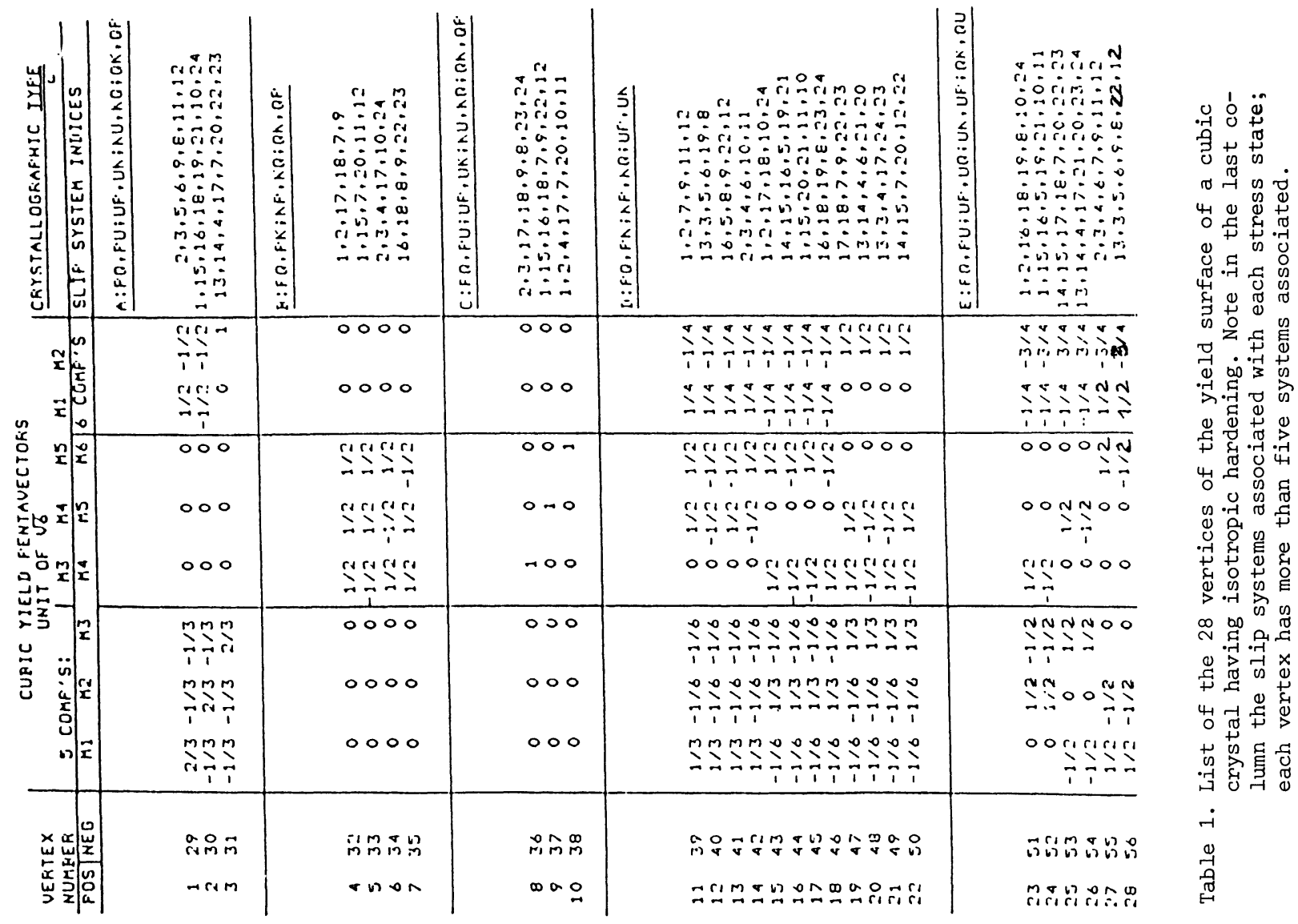


뭉

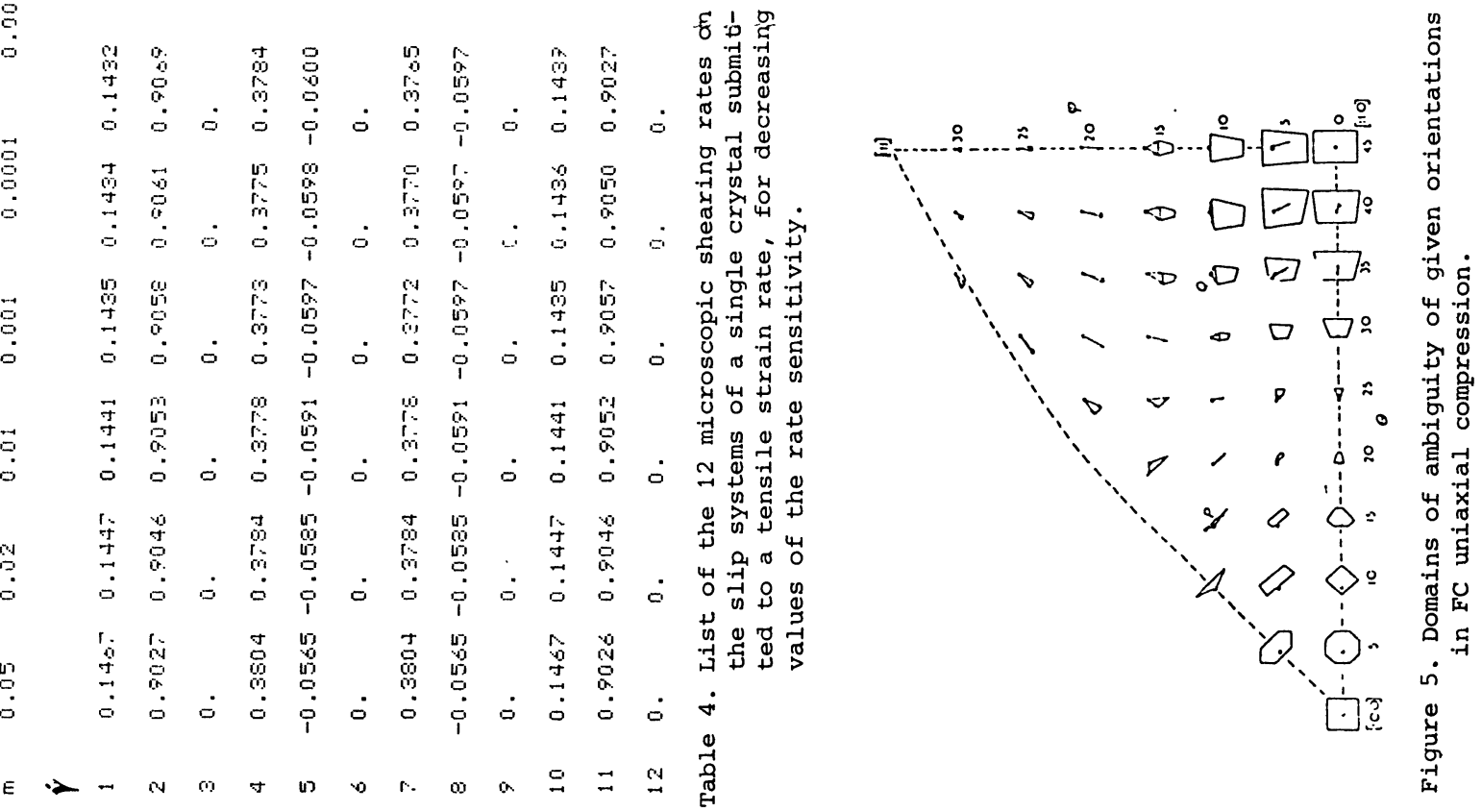

\begin{tabular}{|c|c|c|c|c|c|c|}
\hline 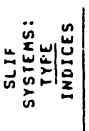 & & 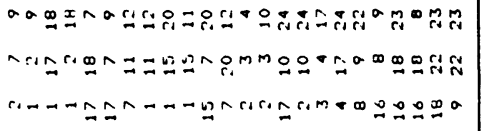 & $\mid \begin{array}{l}\leq \\
0\end{array}$ & \multicolumn{3}{|c|}{ 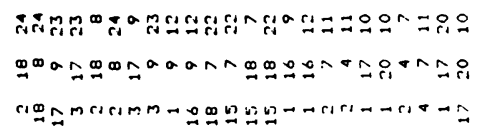 } \\
\hline 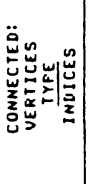 & & 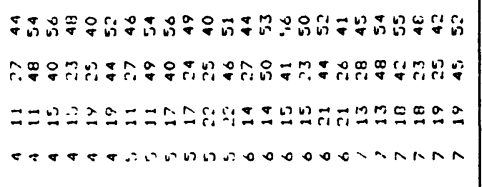 & \multicolumn{4}{|c|}{ 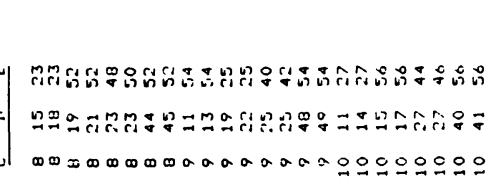 } \\
\hline 峛官 & लا & 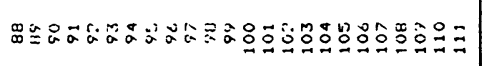 & \multicolumn{4}{|c|}{ 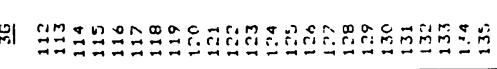 } \\
\hline 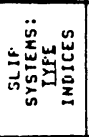 & & 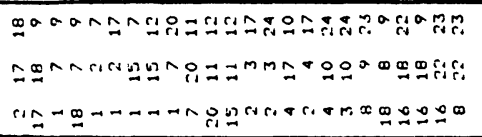 & $\mid \begin{array}{l}\mid \\
\vdots \\
\vdots \\
\end{array}$ & \multicolumn{3}{|c|}{ 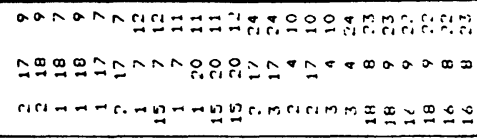 } \\
\hline 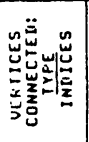 & & 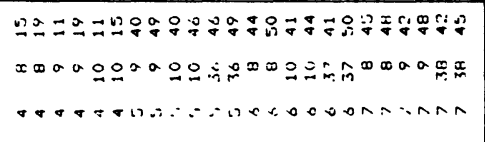 & $\mid \begin{array}{l}0 \\
0\end{array}$ & \multicolumn{3}{|c|}{ 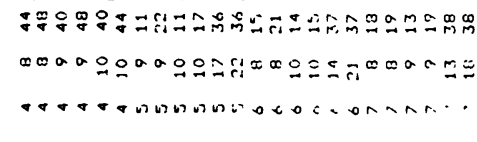 } \\
\hline$\dot{\underline{n}} \mid \dot{z}$ & ثेl. & 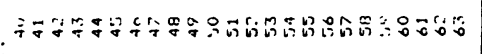 & إس & \multicolumn{3}{|c|}{ 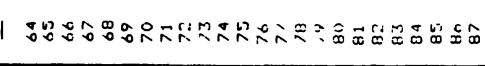 } \\
\hline 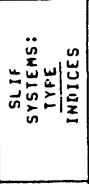 & $\begin{array}{l}s \\
5 \\
c \\
c\end{array}$ & 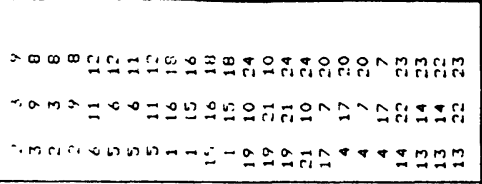 & $\mid \begin{array}{l}5 \\
\dot{c} \\
\dot{c} \\
\mathrm{~d} \\
\mathrm{i}\end{array}$ & $\begin{array}{l}a=0 \\
\operatorname{anc} a \\
\sin = \\
\operatorname{man}\end{array}$ & i & 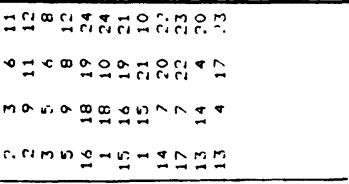 \\
\hline 焉 & \begin{tabular}{r|r}
- \\
- \\
-1
\end{tabular} & 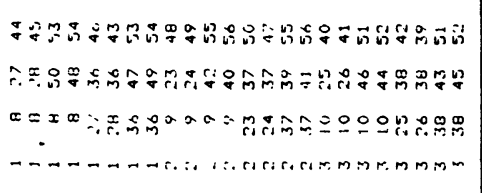 & $\omega$ & 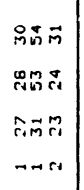 & $\mid$\begin{tabular}{|c|}
$\omega$ \\
$c$
\end{tabular} & 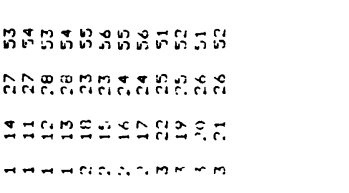 \\
\hline ij & $\leq 1$ & 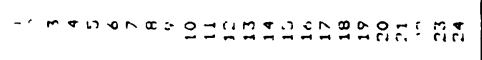 & & mirici & & 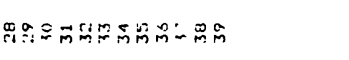 \\
\hline
\end{tabular}

证 

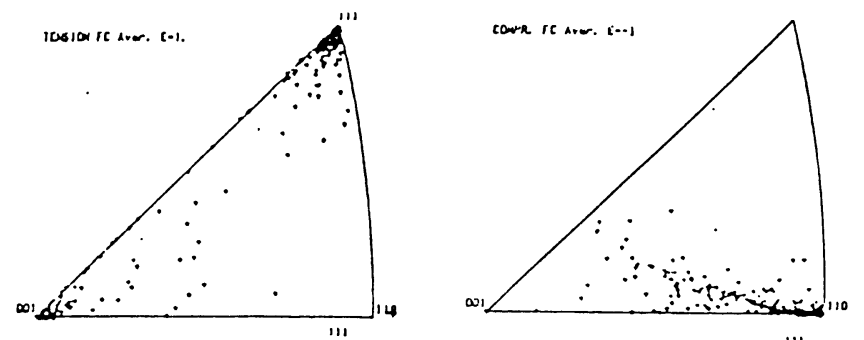

Sochs: $-\sigma_{x x}=\sigma_{2 x}$

(a)
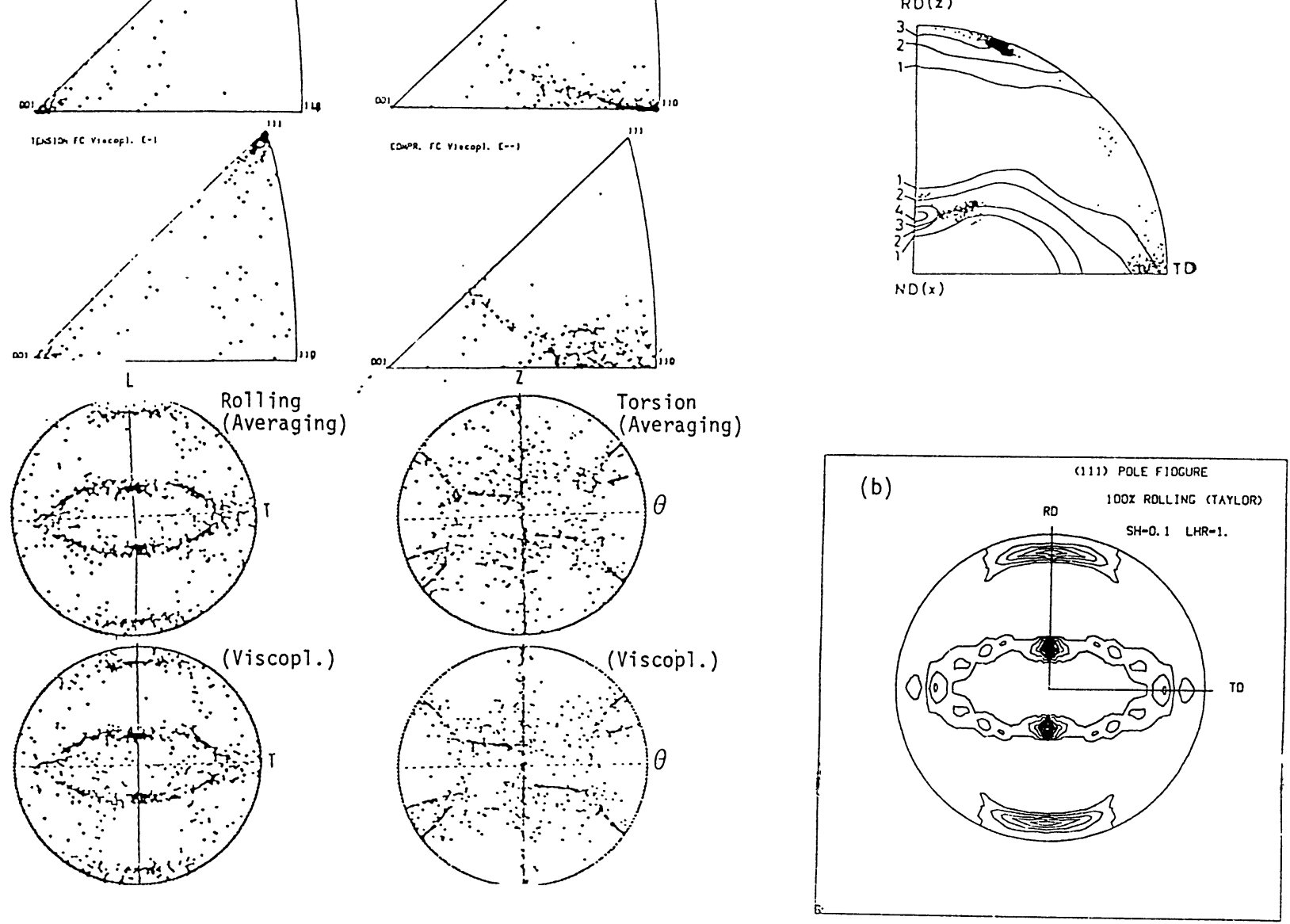

Figure 6. Differences in the FC texture predictions obtained using the averaging technique and visconlasticity.
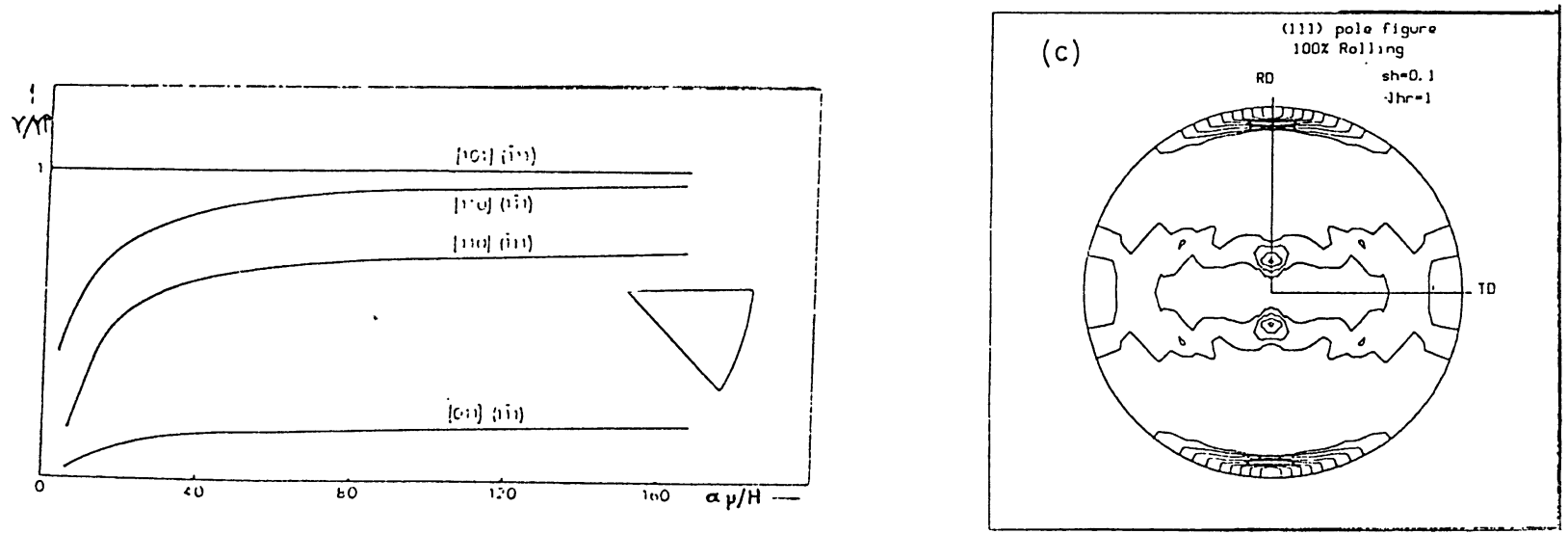

Figure 7. Slip system activity related to the primary system as a function of the parameter $\times \mathrm{H} / \mathrm{H}$. In e.g. stage II, the parameter equals 250 for $\alpha=1$, and lies in the transition region for low (but realistic) values of $\alpha$.

Figure 8. Rolling texture predicted by the viscoplas tic self-consisted scheme (c). Note the $a b$ sence of the Taylor compoment. Note also the difference with Sachs (a) and Taylor (b) pre dictions.

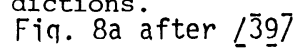



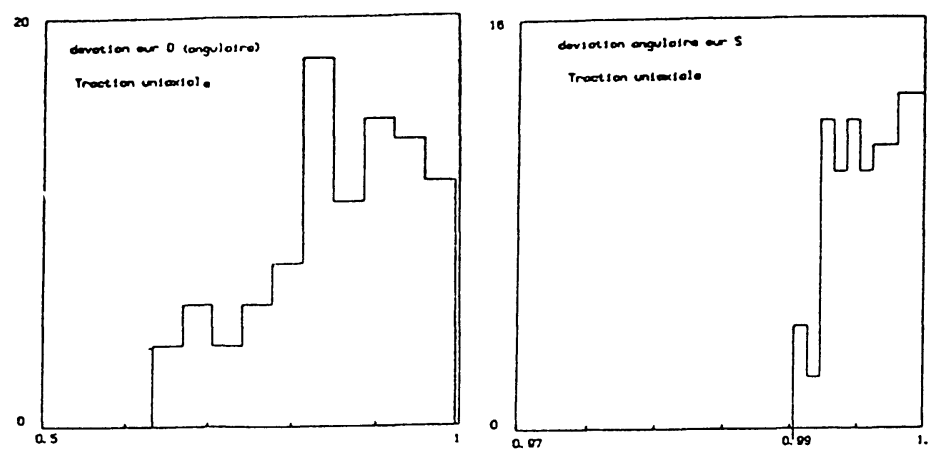

65
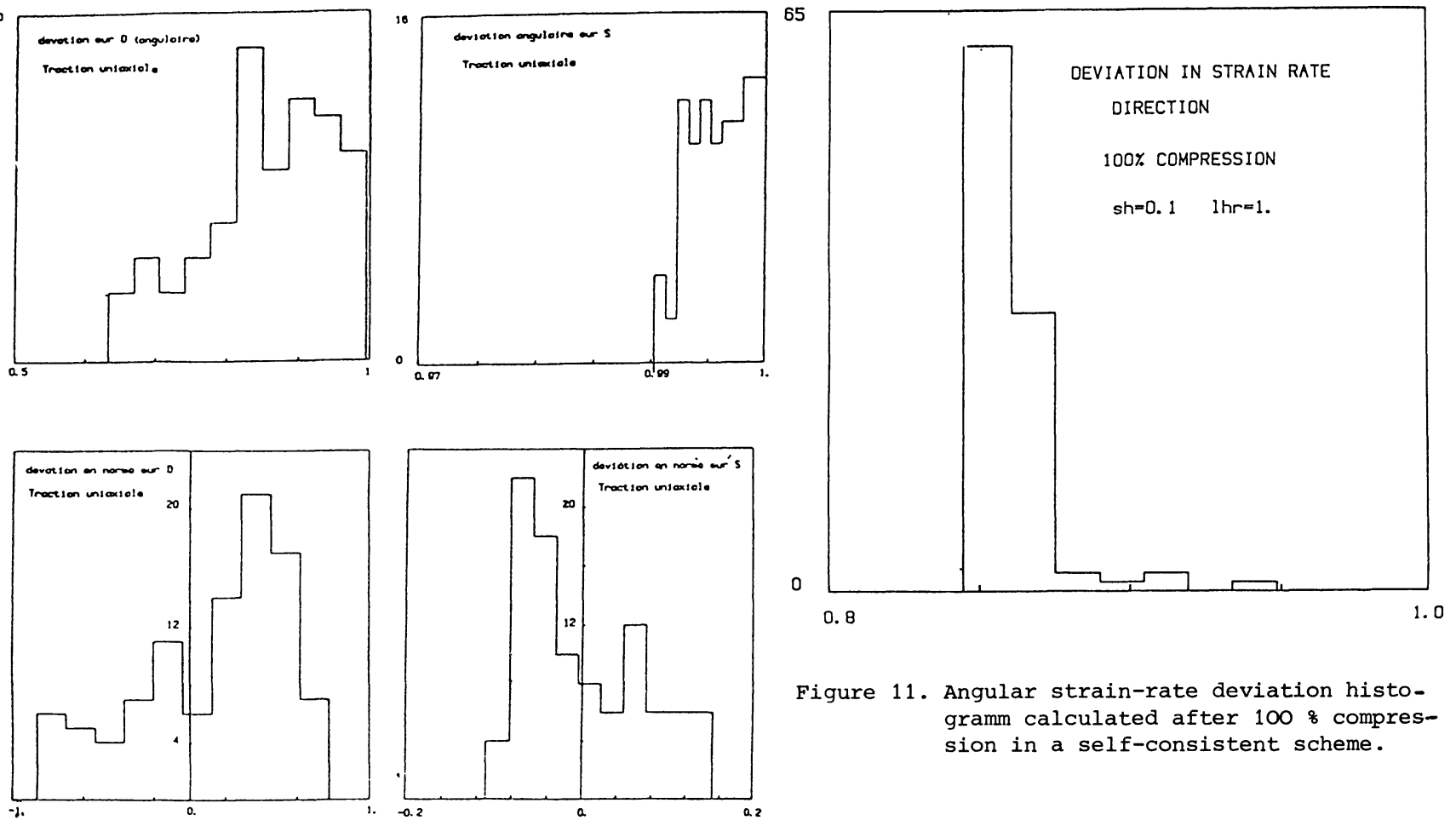

Figure 9. Statistics showing deviations in angle and norm of the strain-rate and the stress w.r.t the corresponding macroscopic values. The stress is much closer to the macroscopic stress than the strain-rate to the average one.

(a) 100: COMJEEcsisn (TAYLOS) $\mathrm{SH}=0.1$ $S H=0.1$
$-H R=1$.

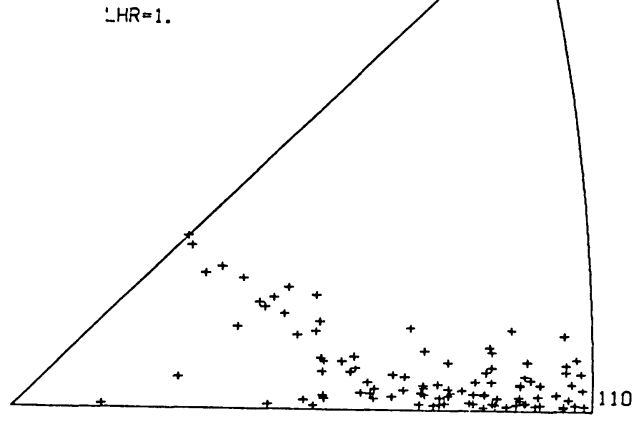

(b)

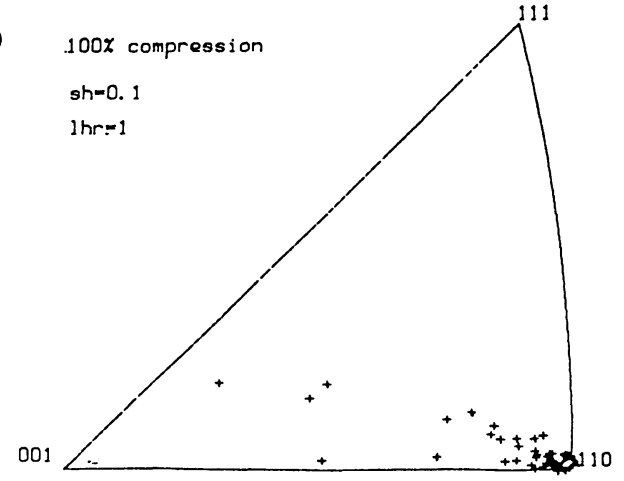

Figure 10. Comparisons of texture predictions in uniaxial compression obtained by the Taylor model (a) and the viscoplastic self-consis tent theory (b).

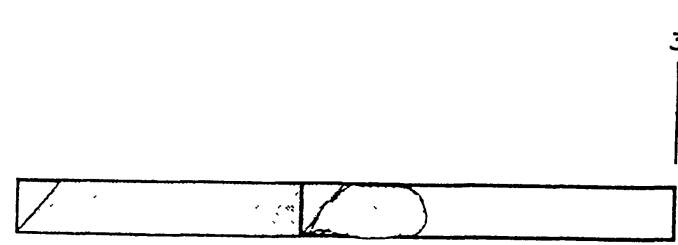

consistent scheme.

Figure 12. Grain shape effect on the misfit strain accomodation.
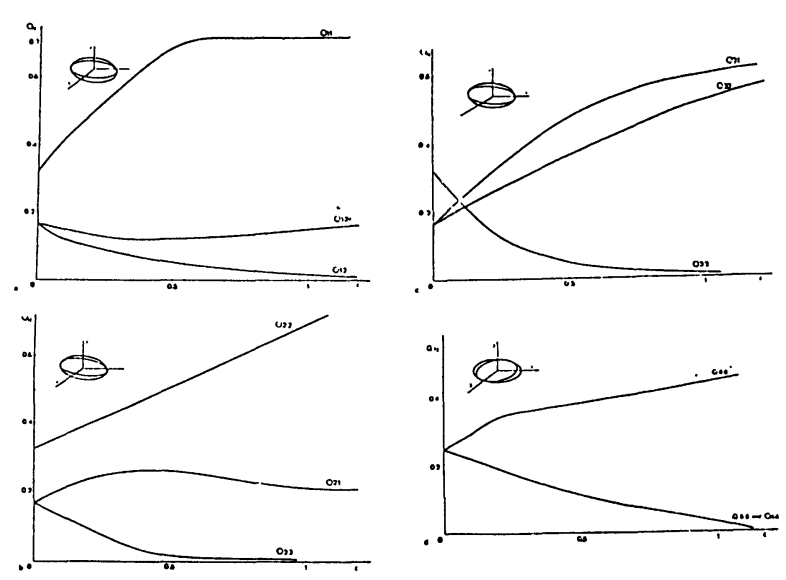

Figure 13. Evolution of the reaction stress moduli in rolling as a function of strain. In particular note the decrease in $Q_{55}$ and $Q_{66}$ ' i.e. $Q_{1313}$ and $Q_{2323}$. 


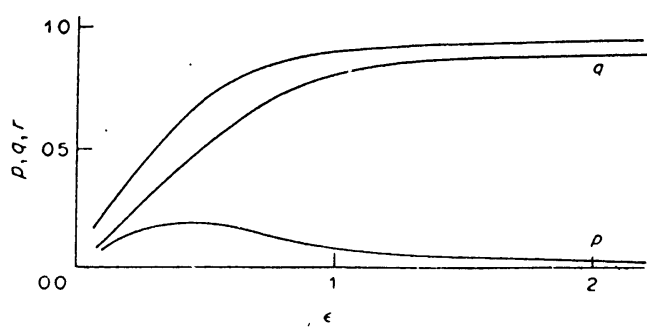

Figure 14. Evolution of the parameters $p, q, r$, used to determine the local total rotation. The RC limit is 1 .
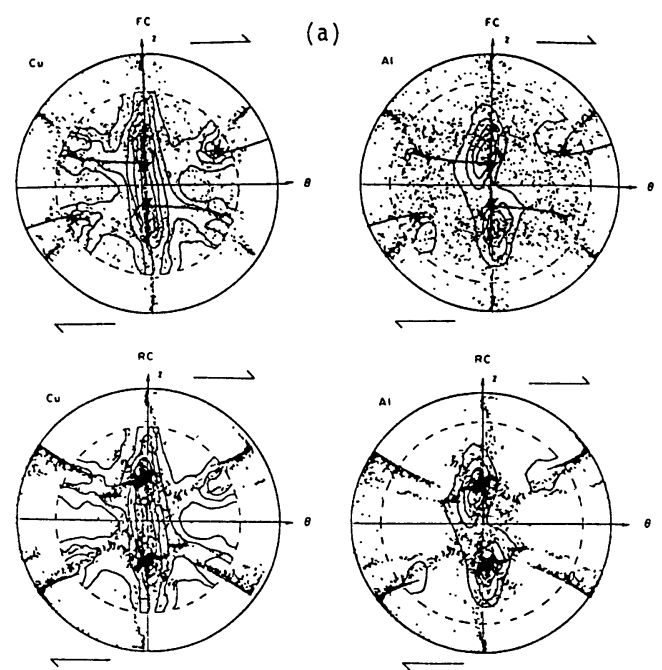

$r \cdot \theta 1$

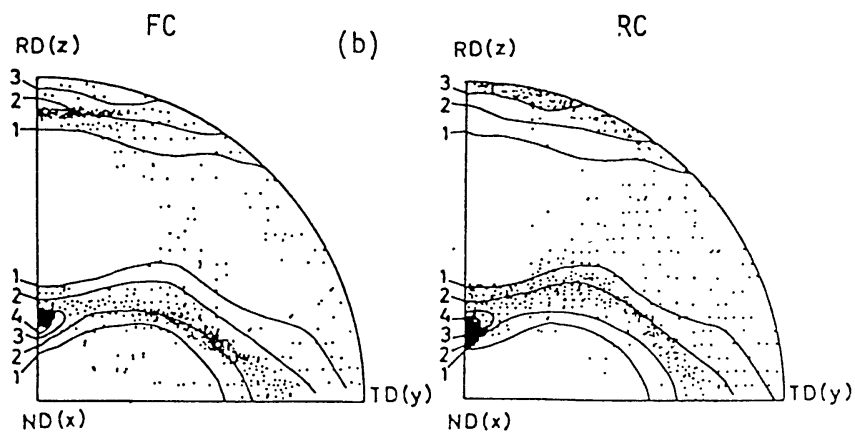

Figure 15. Improvements obtained using grain shape arguments in torsion (a) and rolling (b). The (111) pole figures are shown in FC and RC conditions (dots) and compared with experimental measurements (lines).

Fig. $15 b$ after ref. [3̊2].

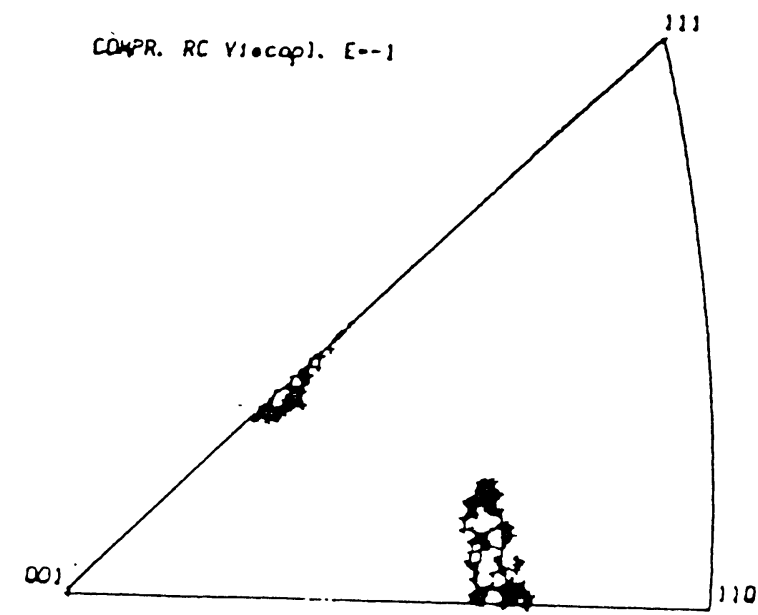

Figure 16. RC textures prediction in uniaxial compression. The real texture is a (110) fiber.

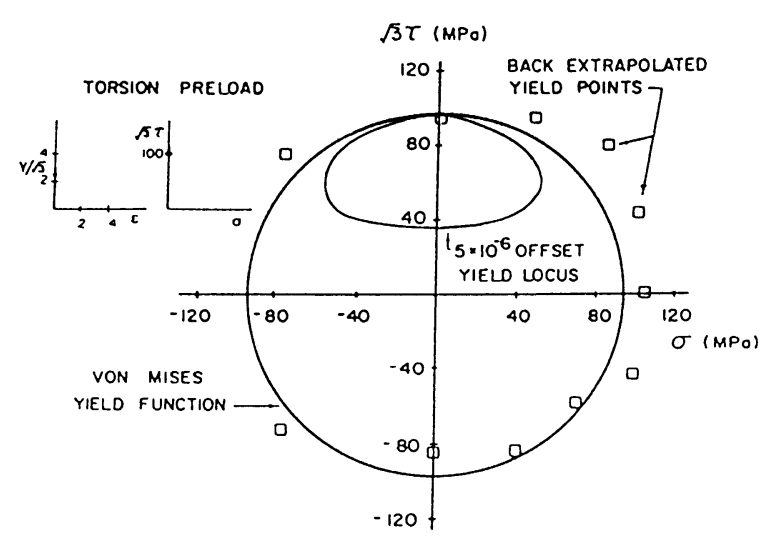

Figure 17. Yield surfaces obtained experimentally on a torsion prestrained material at different offset strains. Note the internal stress effect at a small offset.

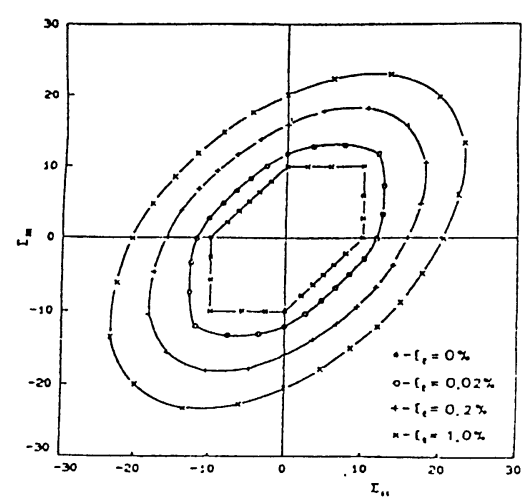

Figure 18. Predicted yield surfaces at different offset strains on a isotropic material without prestraining obtained by an elastoplastic self-consistent scheme. 

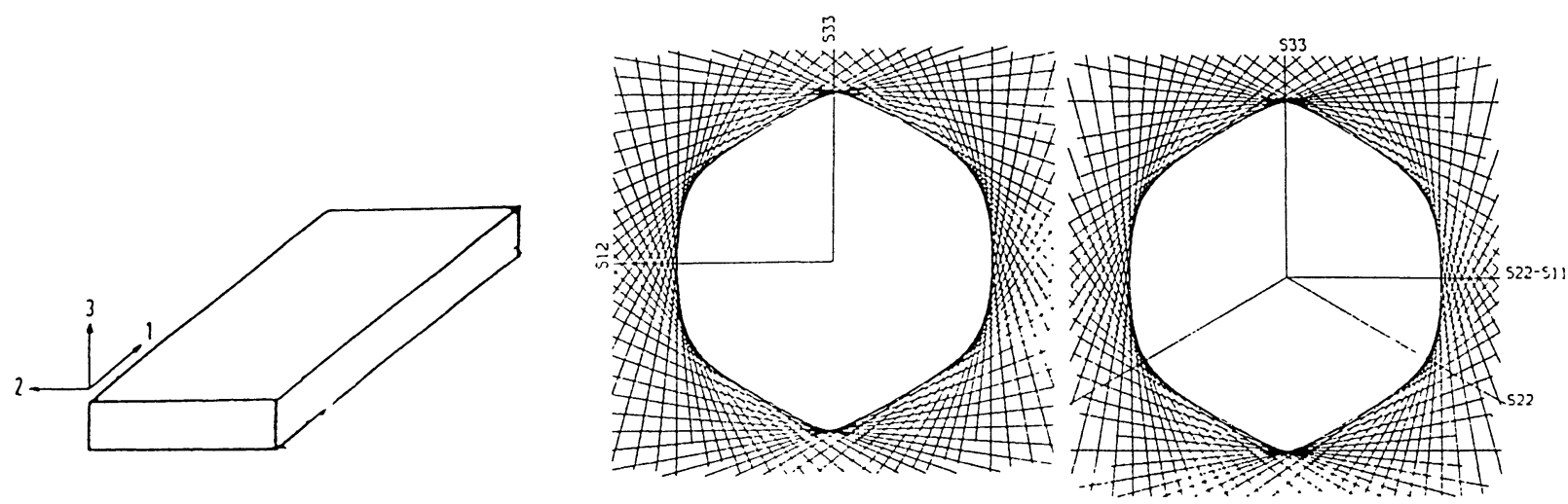

Figure 19. Yield surface of an isotropic material having flat grains. The grain axes are shown in the upper drawing.
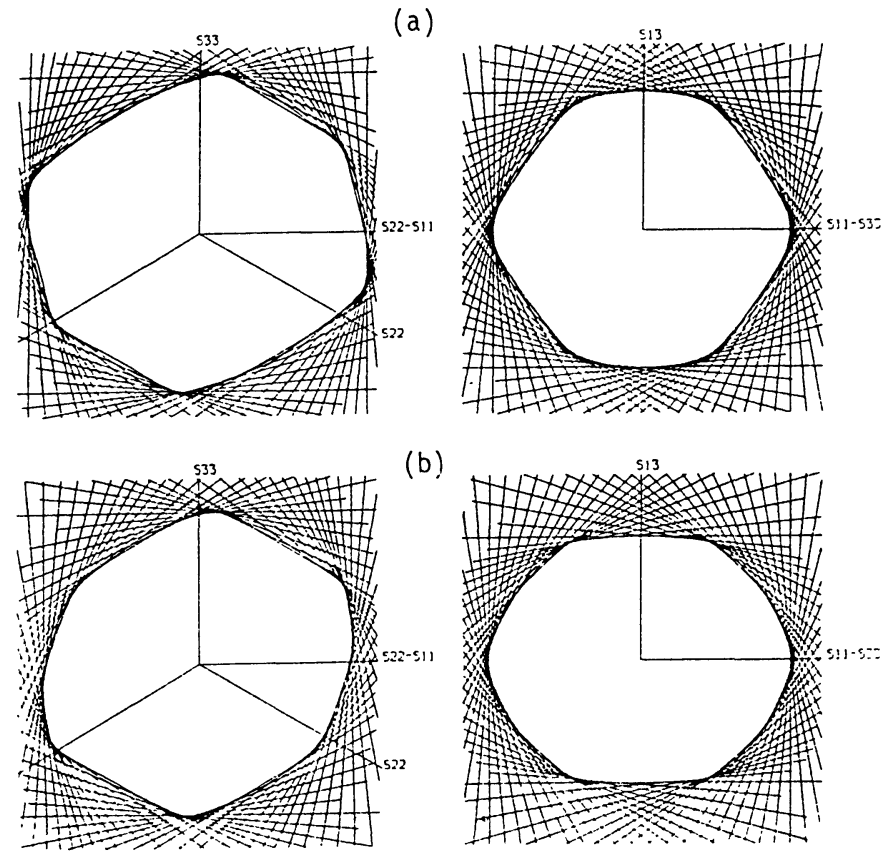

Figure 20. Yield surfaces obtained using a texture material having (a) equiaxed grains

(b) flat grains. 